\title{
PUBLICAÇÕES DOS DOCENTES DO DEPARTAMENTO DE FILOSOFIA
}

2002-2007

\section{José Barata-Moura}

Da Mentira. Um ensaio - transbordante de errores, Lisboa, Caminho, 2007. O Outro Kant, Lisboa, Centro de Filosofia da Universidade de Lisboa, 2007. Kant e o conceito de Filosofia, Lisboa. Centro de Filosofia da Universidade de Lisboa, 2007.

\section{Cristina Beckert}

Ética Ambiental, uma ética para o futuro (coordenação), Lisboa, Centro de Filosofia da Universidade de Lisboa, 2003.

Éticas e Políticas Ambientais (coordenação, com Maria José Varandas), Lisboa, Centro de Filosofia da Universidade de Lisboa, 2003.

Lévinas entre nós (coordenação), Lisboa, Centro de Filosofia da Universidade de Lisboa, 2006.

Hannah Arendt. Luz e Sombra (coordenação, com Maria Luísa Ribeiro Ferreira e Margarida Amaral), Centro de Filosofia da Universidade de Lisboa, 2007.

\section{Paulo Alexandre Esteves Borges}

Pensamento Atlântico, Lisboa, Imprensa Nacional-Casa da Moeda, 2002.

O Budismo e a Natureza da Mente (com Matthieu Ricard e Carlos João Correia, Lisboa, Mundos Paralelos, 2005.

Agostinho da Silva. Uma Antologia, Lisboa, Âncora Editora, 2006.

Tempos de Ser Deus. A espiritualidade ecuménica de Agostinho da Silva, Lisboa, Âncora Editora, 2006.

O Buda e o Budismo no Ocidente e na Cultura Portuguesa (organizador, com Duarte Braga), Lisboa, Ésquilo, 2007.

"O Budismo, uma proximidade do Oriente: ecos, sintonias e permeabilidades no pensamento português", Revista Lusófona de Ciência das Religiões, 
Ano VI, n. ${ }^{\circ} 11$ (Lisboa, 2007) (organizador, com Duarte Braga, do referido dossier temático).

Línguas de Fogo. Paixão, Morte e Iluminação de Agostinho da Silva, Lisboa, Ésquilo, 2006 (Romance).

Folia. Mistério de Pentecostes em três actos, Lisboa, Ésquilo, 2007 (Teatro).

Dalai Lama, Palavras do Coração, Lisboa, Presença, 2003 (com Conceição Gomes) (Tradução).

Tsangyang Gyatso, VI Dalai Lama, Cantos de Amor, Lisboa, Mundos Paralelos, 2005 (Tradução).

Dalai Lama, Uma Mente Tranquila, Lisboa, Editorial Presença, 2006 (com Conceição Gomes) (Tradução).

Padmasambhava, Livro Tibetano dos Mortos, Lisboa, Ésquilo, 2006 (com Rui Lopo) (Tradução).

Dalai Lama / Howard C. Cutler, Serenidade e Realização na Vida Profissional, Lisboa, Editorial Presença, 2007 (Tradução).

Shantideva, A Via do Bodhisattva, Lisboa, Ésquilo, 2007 (que também gravou em áudio-livro, na mesma editora e no mesmo ano) (Tradução).

Patrul Rinpoche, O Caminho da Grande Perfeição, Lisboa, Ésquilo, 2007 (Tradução).

\section{Carlos João Correia}

A Mente, a Religião e a Ciência, ed. de Carlos João Correia, Lisboa, Centro de Filosofia da Universidade de Lisboa, 2003.

Mitos e Narrativas. Ensaios sobre a Experiência do Mal, Lisboa, Centro de Filosofia da Universidade de Lisboa, 2003.

O Budismo e a Natureza da Mente (em colaboração com Matthieu Ricard e Paulo Borges), Lisboa, Mundos Paralelos, 2005.

Jogos de Estética - Jogos de Guerra. 1. ${ }^{\circ}$ Simpósio Nacional de Teoria Estética e Filosofia da Arte. Edição em conjunto com Carlos Couto Sequeira e Costa, José Augusto-França e Levi António Malho, Lisboa, Edições Colibri - Fundação das Casas de Fronteira e Alorna, 2006.

Pensar Azul: Filosofia - $10^{\circ}$ ano (em colaboração com José Arêdes, Fátima Alves e José Carvalho), Texto Editores, Lisboa, 2007.

Arte, Metafisica e Mitologia. Colóquio Luso-Alemão de Filosofia, ed. de Carlos João Correia e Markus Gabriel, Lisboa, CFUL, 2007/2008. 


\section{Maria Luísa Ribeiro Ferreira}

Razão e Paixão. O Percurso de um Curso, Lisboa, Fundação Calouste Gulbenkian, Textos Universitários de Ciências Sociais e Humanas, 2002.

Uma Suprema Alegria. Escritos sobre Espinosa, Coimbra, Quarteto, 2003.

As teias que as mulheres tecem (org.), Lisboa, Edições Colibri, 2003.

Diálogo e controvérsia na modernidade pré-crítica, Lisboa, Centro de Filosofia da Universidade de Lisboa, 2005.

Hannah Arendt. Luz e Sombra (com Cristina Beckert e Margarida Amaral), coordenação do volume das Actas do Colóquio sobre Hannah Arendt, Lisboa, Centro de Filosofia da Universidade de Lisboa, 2007.

\section{António Pedro Mesquita}

Platão e o Problema da Existência, Lisboa, Centro de Filosofia da Universidade de Lisboa, 2003.

Aspectos Disputados da Filosofia Aristotélica, Lisboa, Imprensa Nacional Casa da Moeda, 2004.

Introdução Geral às Obras Completas de Aristóteles, Lisboa, Imprensa Nacional - Casa da Moeda, 2005.

Introdução ao Estudo da Filosofia Antiga, Lisboa, Edições Colibri, 2006.

Liberalismo, Democracia e o Contrário. Um Século de Pensamento Político em Portugal (1820-1930), Lisboa, Sílabo, 2006.

Vida de Aristóteles, Lisboa, Sílabo, 2006.

O Pensamento Político Português no Século XIX. Uma Síntese Histórico-Crítica, Lisboa, Imprensa Nacional - Casa da Moeda, 2006.

Salazar na História Política do seu Tempo. Um Estudo Ideográfico sobre o Perfil Doutrinário do Regime Salazarista, Lisboa, Editorial Caminho, 2007.

\section{Leonel Ribeiro dos Santos}

Antero de Quental, Uma visão moral do mundo, Lisboa, Imprensa Nacional-Casa da Moeda, 2002.

Linguagem, Retórica e Filosofia no Renascimento, Lisboa, Edições Colibri, Lisboa, 2004.

Kant 2004: Posteridade e Actualidade, Actas do Colóquio do mesmo nome, realizado em Novembro de 2004, Centro de Filosofia da Universidade de Lisboa, Lisboa, 2006 (organizador).

O Espirito da Letra. Ensaios de Hermenêutica da Modernidade, Imprensa Nacional-Casa da Moeda, Lisboa, 2007. 
Filosofia Kantiana do Direito e da Política, Actas do Seminário com o mesmo nome, Centro de Filosofia da Universidade, Lisboa, 2007 (organizador).

Kant em Portugal: 1974-2004, Centro de Filosofia da Universidade, Lisboa, 2007 (organizador).

Schiller, Cidadão do Mundo, Actas do Colóquio com o mesmo nome, Centro de Filosofia da Universidade, Lisboa, 2007 (organizador).

\section{Carlos Couto S.C.}

Caderno De Demónios - Os Últimos Anos De Nietzsche Ou A Ilha Dos Mortos, Fenda Lisboa, 2002.

Três Lições De Arquitectura - Ensaio De Filosofia, Fenda 2002.

Nietzs / Che - A Ilha Do Sepulcro (Peça-Monólogo), A.Penas, Lisboa 2003.

Con-Ficções, A.Penas, Lisboa, 2003.

Adamitié - Nocturnos, Porto 2004 (Homenagem a Adami).

a deus, Fenda, Lisboa 2004

Nota Breve Sobre Bresson, In Bresson/Notas Sobre O Cinematógrafo (trad. Pedro Mexia), Lisboa, 2004.

Jogos De Estética-Jogos De Guerra, Colóquio I /Estéticas (co-organizador), Lisboa, Colibri 2005.

Vedutismo - Estéticas, Pé-De-Página, Coimbra, 2005.

Estéticas E Filosofias - Controvérsias Para O Próximo Milénio, colóquio (co-organizador), Lisboa, 2005

Manuelinos \& Franciscanos, A.Penas, 2006.

Rta, Mon Chat, Lisboa, A.Penas 2007.

\section{Adriana Veríssimo Serrão}

Ludwig Feuerbach, A Essência do Cristianismo. Lisboa, Fundação Calouste Gulbenkian, 2. ${ }^{\text {a }}$ ed.: 2002 (tradução).

A Invenção do "Homem". Raça, Cultura e História na Alemanha do século XVIII (em co-autoria com Manuela Ribeiro Sanches). Lisboa, Centro de Filosofia da Universidade de Lisboa, 2002.

Ludwig Feuerbach. Filosofia da sensibilidade. Escritos (1841-1846). Lisboa, Centro de Filosofia da Universidade de Lisboa, 2004 (tradução).

Pensar a Sensibilidade: Baumgarten - Kant - Feuerbach, Lisboa, Centro de Filosofia da Universidade de Lisboa, 2007.

\section{Maria Leonor Xavier}

Questões de Filosofia na Idade Média (Forum de Ideias; 27), Lisboa, Edições Colibri, 2007. 


\section{CENTRO DE FILOSOFIA DA UNIVERSIDADE DE LISBOA}

\section{PUBLICAÇÕES E ACTIVIDADES}

\section{PUBLICAÇÕES}

\section{PUBLICAÇÕES PRÓPRIAS}

Do Ponto de Vista do Universo, autoria de Pedro Galvão, Academica 7, Centro de Filosofia da Universidade de Lisboa, 2008.

Arte, Metafísica e Mitologia, coordenação de Carlos João Correia e Markus Gabriel, Acta 7, Lisboa, Centro de Filosofia da Universidade de Lisboa, $2007 / 2008$.

A Morte e a Origem. Em torno de Heidegger e Freud, coordenação de Irene Borges-Duarte, Acta 8, Lisboa, Centro de Filosofia da Universidade de Lisboa, 2008.

\section{PARCERIAS}

Convergências \& Afinidades. Homenagem a António Braz Teixeira, CEFi e Centro de Filosofia da Universidade de Lisboa, 2008. 
Aristóteles Obras Completas, Vol. IV Tomo II: História dos Animais, Autoria de Aristóteles, tradução de Maria F. S. Silva, IN-CM e Centro de Filosofia da Universidade de Lisboa, 2008.

Teologia Mística, tradução e Introdução de Maria Leonor Xavier, Ésquilo e Centro de Filosofia da Universidade de Lisboa, 2008.

Lógica. A Pergunta pela Essência da Linguagem, autoria de Martin Heidegger, tradução de Maria A. Pacheco e Helga H. Quadrado, Fundação Calouste Gulbenkian e Centro de Filosofia da Universidade de Lisboa, 2008.

\section{PUBLICAÇÕES PERIÓDICAS}

Disputatio - International Journal of Philosophy, Vol. II n. ${ }^{\circ}$ 22, Edição João Branquinho, Centro de Filosofia da Universidade de Lisboa, 2007.

\section{EVENTOS DE NATUREZA CIENTÍFICA E CULTURAL}

\section{COLÓQUIOS E JORNADAS}

Congresso Internacional «Delfim Santos e a Escola do Porto», 17 a 19 de Março 2007, UTAD/FLUP/FLUL/FCG, organização de António Braz Teixeira, Celeste Natário, Cristiana de Soveral, Maria L. Sirgado Ganho e Renato Epifânio.

Congresso Internacional «Still Reading Hegel: after 200 years of the "Phenomenology of Spirit"», 19 de Novembro 2007, FLUC, organização de Edmundo Balsemão Pires.

III Congresso da Associação Portuguesa de Filosofia Fenomenológica: «Mundo da Vida, Racionalidade, Ciência», «A Fenomenologia Luso-Brasileira», 5 a 8 de Dezembro 2007, FLUL, organização de Pedro Alves.

2. ${ }^{a}$ Reunião Plenária da Sociedade Ibérica de Filosofia Grega, 29 Fevereiro 2008, FLUL, coordenação de António Pedro Mesquita.

Encontro com Jean Leclercq. Fenomenologia da Vida, 17 e 18 de Abril 2008, ESEL/FLUL, coordenação de Florinda Martins.

\section{SEMINÁRIOS E CONFERÊNCIAS}

Seminários do Projecto PTDC/FIL/64836/2006 «Filosofia, Medicina e Sociedade», Faculdade de Letras da Universidade de Lisboa, Centro de Filosofia da Universidade de Lisboa, coordenação de Adelino Cardoso. 
Seminários do Projecto PTDC/FIL/64249/2006 «A Questão de Deus. História e Crítica», Faculdade de Letras da Universidade de Lisboa, Centro de Filosofia da Universidade de Lisboa, coordenação de Maria Leonor Xavier.

Seminários do Projecto POCI/FIL/60600/2006 «Heidegger em Português», Universidade de Évora, Centro de Filosofia da Universidade de Lisboa, coordenação de Irene Borges-Duarte.

Seminário Permanente de Filosofia Analítica, Faculdade de Letras da Universidade de Lisboa, Centro de Filosofia da Universidade de Lisboa, coordenação de João Branquinho.

\section{PUBLICAÇÕES E EVENTOS DE NATUREZA CIENTÍFICA E CULTURAL PREVISTOS PARA 2008}

\section{PUBLICAÇÕES PRÓPRIAS}

Formas de Arte. A Prática Crítica de Berlioz, Kierkegaard, Liszt e Schumann, autoria de Elisabete Sousa, Academica 8, Centro de Filosofia da Universidade de Lisboa, 2008.

Um pensar para o Outro. Estudos sobre Emmanuel Lévinas, autoria de Cristina Beckert, Studia 4, Centro de Filosofia da Universidade de Lisboa, 2008.

Varia Antiqua. Estudos de Filosofia Antiga, autoria de António Pedro Mesquita, Studia 5, Centro de Filosofia da Universidade de Lisboa, 2008.

\section{EVENTOS CIENTÍFICOS PARA 2008}

Workshop «On Negation and Denial», Faculdade de Letras da Universidade de Lisboa, Julho de 2008.

Seminário Permanente de Filosofia Analítica, Faculdade de Letras da Universidade de Lisboa, Centro de Filosofia da Universidade de Lisboa, coordenação de João Branquinho.

Seminários do Projecto PTDC/FIL/64836/2006 «Filosofia, Medicina e Sociedade», Faculdade de Letras da Universidade de Lisboa, Centro de Filosofia da Universidade de Lisboa, coordenação de Adelino Cardoso.

Seminários do Projecto PTDC/FIL/64249/2006 «A Questão de Deus. História e Crítica», Faculdade de Letras da Universidade de Lisboa, Centro de Filosofia da Universidade de Lisboa, coordenação de Maria Leonor Xavier. 
Nota: os preços dos livros estão indicados na página do Centro de Filosofia na internet: www.centrofilosofia.org

Centro de Filosofia da Universidade de Lisboa

Faculdade de Letras de Lisboa

Alameda da Universidade

P-1600-214 Lisboa

Tel/Fax: 217920091 •E-mail: c.filosofia@ffl.ul.pt

Web: www.centrofilosofia.org

N. ${ }^{\circ}$ Id. Fiscal: 502447699

Centro de Filosofia da Universidade de Lisboa, 13 de Abril de 2007. 


\title{
DEPARTAMENTO DE FILOSOFIA DA UNIVERSIDADE DE LISBOA
}

\author{
Estudos Pós-Graduados em Filosofia - 2008-2009
}

SEMINÁRIOS DE MESTRADO $\left(2 .{ }^{\circ}\right.$ Ciclo $)$

O programa de mestrado em Filosofia consiste em:

- 6 Unidades Curriculares (UC) de Tópicos de Filosofia e 2 Unidades Curriculares (UC) de Opção, a completar nos dois primeiros semestres.

- 2 UC de Seminários de Investigação, a completar nos terceiro e quatro semestres.

\section{- Dissertação de Mestrado.}

As UC de Tópicos de Filosofia encontram-se repartidas por seis grupos: História da Filosofia; Filosofia da Linguagem e da Mente; Filosofia do Espaço Público; Filosofia da Natureza, da Vida e do Ambiente; Estética e Filosofia da Arte; Epistemologia e Metafísica. As UC de Opção podem ser obtidas em qualquer programa da Universidade de Lisboa. Os Seminários de Investigação destinam-se a apoiar a investigação conducente à dissertação de mestrado.

\section{Primeiro Semestre}

- Antropologia Filosófica Contemporânea - (História da Filosofia I) Adriana Veríssimo Serrão - 4. . feira, $18-22 \mathrm{~h}$

Salvaguardar a indivisibilidade do ser-humano e, ao mesmo tempo, captar a complexidade da sua existência são os pólos característicos do modo contemporâneo de colocar a questão do Homem. O seminário visa o aprofundamento do problema a partir dos seguintes tópicos: a) Kant: a dualidade da razão e do homem ou a tensão entre filosofia transcendental e antropologia pragmática. b) L. Feuerbach: os paradoxos da integralida- 
de na dinâmica entre essência e existência. c) Georg Simmel: o homem como "ponte e porta" ou o conflito entre a vida e as formas; d) Helmuth Plessner: da posicionalidade excêntrica aos limites da expressividade.

- Bioética: Fundamentos Filosóficos - (Filosofia do Espaço Público I) Cristina Beckert $-5 .^{\text {a }}$ feira, $18-22 \mathrm{~h}$

O presente seminário de pós-graduação pretende, como o título indica, perscrutar os fundamentos filosóficos da Bioética, a diversos níveis. Assim, procurar-se-á reportar os diferentes modelos bioéticos (principialista, utilitarista, libertário, das virtudes e outros) às teorias da ética clássica, bem como questionar alguns conceitos filosóficos, como os de pessoa, vida, liberdade, responsabilidade, no confronto com o desenvolvimento tecnocientífico contemporâneo.

- Luz da Razão: Para uma Fundamentação Onto-metafísica - (Epistemologia e Metafísica I) - Mafalda Blanc - 6. ${ }^{\mathrm{a}}$ feira, 16 - 20h

Dos fundamentos intuitivos da razão clássica ao construtivismo da razão moderna. O carácter problemático da racionalidade científica: conjecturas, refutações e relativismo. A crítica da onto-teologia e o momento kanteano da humanação da razão. Insuficiência dos formalismos e do idealismo tanscendental face aos requisitos ontológicos da verdade. Necessidade de uma refundação onto-metafísica do pensar e a possibilidade de um regresso ao realismo.

- O Pensamento Político Moderno - (História da Filosofia II) - Leonel Ribeiro dos Santos $-3 .^{\text {a }}$ feira, $14-18 \mathrm{~h}$

- O Pensamento da Arte em Deleuze - (Estética e Filosofia da Arte I) Nuno Nabais -2 . $^{\text {a }}$ feira, $14-18 \mathrm{~h}$

- Ética Pública - (Filosofia do Espaço Público II) - Viriato Soromenho-Marques -3 . $^{\text {a }}$ feira, $18-22 \mathrm{~h}$

A tese central deste curso procurará demonstrar-se que a ética pode ser identificada como um "motor invisível", mas fundamental, na oscilante marcha histórica da razão prática. A ética é sempre pública nas suas consequências e universal nos seus fundamentos, sendo a única esfera da racionalidade prática que permite, nos momentos de convulsão histórica, a reconstrução do triângulo prático constituído pelos vértices da experiência moral, do ordenamento jurídico e das instituições políticas. O tema da ética pública será objecto de uma análise a partir de seis temas centrais, para os quais serão convocados textos e autores fundamentais da tradição ética e política do Ocidente.

- Seminário de Investigação - Carlos João Correia - 5. ${ }^{\mathrm{a}}$ feira, 20 - 22h

Este seminário tem como objectivo criar as condições para que estudantes pós-graduados possam determinar as linhas fundamentais, tanto 
temáticas, como metodológicas, das suas dissertações. Para esse efeito, os participantes do seminário farão periodicamente apresentações do material produzido, ao mesmo tempo que se discutirão questões fundamentais de índole filosófica consensualmente consideradas como pertinentes.

\section{Segundo Semestre}

- Ciência e Teoria da Ciência na Antiguidade Clássica - (História da Filosofia III) - António Pedro Mesquita - 2. ${ }^{a}$ feira, 14 - $18 \mathrm{~h}$

- O Fantasma da Obra - Topologias do Cinema, da Música e da Pintura - (Estética e Filosofia da Arte II) - Carlos Couto Sequeira Costa - 5. ${ }^{\text {a }}$ feira, $18-22 \mathrm{~h}$

Existe uma postura "fantasmal" (e um $\mathrm{mal}$ ) da Obra, obra de arte e obra filosófica? Partindo do "jogo de linguagem" Fantasma da Obra, procurar-se-à dilucidar, a partir de suportes imagéticos, textuais e musicais (lembremos Mendelssohn 1809-2009, múltiplos), a pregnância mais irredutível e minimal do "espaço da obra" (Blanchot, Agacinsky, Heidegger, Deleuze, Didi-Huberman, outros), abarcando a contemporaneidade filosófica e estética .

- Arte, Metafísica e Mitologia - (Epistemologia e Metafísica II) - Carlos João Correia -2 . $^{\mathrm{a}}$ feira, $18-22 \mathrm{~h}$

O seminário tem como seu principal objectivo analisar questões metafísicas fundamentais, tendo como ponto de referência o modo como elas são abordadas nas obras artísticas e nas produções mitológicas da humanidade. O fio condutor desta análise será o problema metafísico clássico da identidade pessoal à luz da criação artística (literária, musical, plástica) cultivada nessa era de "recusa da desmitologização" (Laurence Coupe), a saber, o modernismo.

- Arte e Fenomenologia - (Estética e Filosofia da Arte III) - Isabel Matos Dias $-4 .^{\mathrm{a}}$ feira, $14-18 \mathrm{~h}$

- Filosofias da Praxis - (Filosofia do Espaço Público III) - José Barata-Moura -3 . $^{\text {a }}$ feira, $20-22,4 .^{\text {a }}$ feira $20-22 \mathrm{~h}$

- Seminário de Investigação - Cristina Beckert - 3. a feira, 18 - 20h

Este seminário tem como objectivo fundamental preparar os estudantes de pós-graduação para a elaboração das respectivas dissertações, tanto do ponto de vista formal como temático. Para tal, serão abordados temas e/ou textos do interesse de todos, escolhidos de comum acordo, e ocorrerão apresentações orais acerca do andamento da investigação dos participantes. 
SEMINÁRIOS DE DOUTORAMENTO $\left(3 .{ }^{\circ}\right.$ Ciclo $)$

\section{A - DOUTORAMENTO EM FILOSOFIA}

O programa de doutoramento em Filosofia consiste em:

- 6 Unidades Curriculares (UC) de Tópicos Avançados de Filosofia, a completar nos três primeiros semestres.

- 3 UC de Seminários de Investigação, a completar nos terceiro, quatro e quinto semestres.

\section{- Dissertação de Doutoramento.}

Para o Curso de Formação Avançada, valem todos os seminários de pós-graduação em Filosofia oferecidos pelo Departamento de Filosofia, desde que integrados em planos de estudos definidos pelo Orientador.

\section{B - DOUTORAMENTO EM CIÊNCIA POLÍTICA}

Primeiro Semestre

- Pensamento Político Antigo e Medieval - José Barata-Moura e Pedro Calafate $-4 .^{\text {a }}$ feira, $16-18 \mathrm{~h} 00$

- Ética Pública - Viriato Soromenho-Marques - 3. ${ }^{\mathrm{a}}$ feira, $18-20 \mathrm{~h} 00$

1. ${ }^{\circ}$ Tópico: Ética Pública e esfera moral: determinação das grandes características da ética; procura da especificidade da ética pública; relações entre ética e política; realismo e idealismo transcendental; a mediação do contratualismo. $2{ }^{\circ}$ Tópico: A ética pública e o campo da acção: A esfera do agir humano na pluralidade das suas dimensões; labor, trabalho e acção; poder e violência; a construção do "espaço público"; a estrutura narrativa da acção.

Segundo Semestre

- Pensamento Político Moderno e Contemporâneo - Leonel Ribeiro dos Santos e António Franco Alexandre - 3. a feira, 16 - 18h 
C - DOUTORAMENTO EM CIÊNCIA COGNITIVA

\section{Primeiro Semestre}

- Introdução à Ciência Cognitiva . João Branquinho - 3. a feira, $15 \mathrm{~h} 30$ $17 \mathrm{~h} ; 5 .^{\mathrm{a}}$ feira $15 \mathrm{~h} 30-17 \mathrm{~h}$

Segundo Semestre

- Filosofia da Mente, Linguagem e Cognição I - João Branquinho - 3. ${ }^{\mathrm{a}}$ feira $15 \mathrm{~h} 30-17 \mathrm{~h} ; 5$. feira, $15 \mathrm{~h} 30-17 \mathrm{~h}$

\section{ACÇÕES DE TRABALHO}

Os cursos oferecidos têm a duração de 16 semanas e podem ser creditados como Acções de Formação para professores do Ensino Secundário. A cada uma destas Acções de Formação corresponde um total de 50 horas equivalentes a 2 créditos.

\section{Director de Estudos Pós-Graduados}

Prof. ${ }^{a}$ Dr. ${ }^{a}$ Adriana Veríssimo Serrão

adrianaserrao@fl.ul.pt

\section{Informações}

Todas as informações podem ser consultadas no sítio da Faculdade de

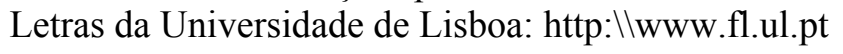

As informações relativas ao Doutoramento em Ciência Política e ao Doutoramento em Ciência Cognitiva podem ser obtidas na Reitoria da Universidade de Lisboa. 